Proyecciones

Vol. $13 N^{\circ} 1$, pp.19-24 Julio 1994

Universidad Católica del Norte

Antofagasta - Chile

\title{
HOMOTOPA DE T-ALGEBRAS DE RANGO 3
}

\author{
RODOLFO BAEZA V. Y RAÚL BENAVIDES G.* \\ Universidad de La Frontera, Temuco-Chile
}

\begin{abstract}
Resumen
En este artículo caracterizamos los idempotentes de la homótopa de la $T$-álgebra $\mathbf{A}=\mathbf{K e} \oplus U \oplus V$ de rango 9 y probamos que si su homótopa también es de rango 3, entonces $\mathbf{A}=\mathbf{K} \boldsymbol{e} \oplus V$.
\end{abstract}




\section{Preliminares}

Dada una álgebra $\mathbf{A}$ y un elemento fijo $a \in \mathbf{A}$, Thedy (ver [4]) construye el álgebra homótopa $\mathbf{A}^{(a)}$ manteniendo la misma estructura lineal de $\mathbf{A}$, pero con la nueva multiplicación:

$$
x \cdot a \cdot y=(x a) y+x(a y)-a(x y)
$$

Evidentemente la conmutatividad de $\mathbf{A}$ implica la conmutatividad de $\mathbf{A}^{(a)}$.

Si $(\mathbf{A}, \omega)$ es una álgelora ponderada y $a \in \mathbf{A}$ es de peso $k \neq 0$, podemos definir la función $\omega^{\prime}: \mathbf{A}^{(a)} \longrightarrow \mathbf{K}$ tal que $\omega^{\prime}(x)=k \omega(x)$. Como $\omega^{\prime}(x \cdot a y)=k \omega(x \cdot a y)=$ $k \omega(x) \omega(a) \omega(y)=k \omega(x) k \omega(y)=\omega^{\prime}(x) \omega^{\prime}(y)$ se tiene que $\left(\mathbf{A}^{(a)}, \omega^{\prime}\right)$ es también un álgebra ponderada. Para el elemento $b=k^{-1} a$ de peso 1 , consideremos el álgebra ponderada $\left(\mathbf{A}^{(b)}, \omega\right)$ y la biyección $\psi:\left(\mathbf{A}^{(a)}, \omega^{\prime}\right) \longrightarrow\left(\mathbf{A}^{(b)}, \omega\right)$ dada por $\psi(x)=k x$. Dado que $\psi$ es lineal, que $\omega(\psi(x))=\omega(k x)=k \omega(x)=\omega^{\prime}(x), \forall x \in \mathbf{A}$ $y$ además que $\psi(x \cdot a y)=k(x \cdot a y)=k((x a) y+x(a y)-a(x y))=k^{2}(x \cdot b y)=$ $(k \cdot x) \cdot{ }_{a}(k y)=\psi^{\prime}(x) \cdot{ }_{a} \psi(y)$, se tiene que $\left(\mathbf{A}^{(a)}, \omega^{\prime}\right) \cong\left(\mathbf{A}^{(b)}, \omega\right)$, (ver González [2]). Totemos que la homótopas producidas por elementos de peso uno no son necesariamente isomorfas, como lo muestra el siguiente ejemplo.

Ejemplo: Sea $\mathbf{A}$ el álgebra $<c_{o}, c_{1}>_{\mathbb{R}}$, cuyo producto está dado por $c_{o}^{2}=$ $c_{0}+c_{1}, c_{0} c_{1}=c_{1} c_{0}=3 c_{1}$ y $c_{1}^{2}=c_{1}$. A es un álgebra ponderada con homomorfismo peso $\omega: \mathbf{A} \longrightarrow \mathbb{R}$ dado por $\omega\left(c_{0}\right)=1 \mathrm{y} \omega\left(c_{1}\right)=0$. Nótese que los únicos idempotentes no nulos de $\mathbf{A}^{\left(c_{0}\right)}$ son $c_{o}-c_{1}$ y $c_{0}-\frac{4}{3} c_{1}$. Además $\mathbf{A}^{\left(c_{0}+c_{1}\right)}$ no tiene idempotentes no nulos. Entonces $\mathbf{A}^{\left(c_{0}\right)} \not \mathbf{A}^{\left(c_{o}+c_{1}\right)}$.

En este trabajo $I(\mathbf{A})$ indicará el conjunto de todos los idempotentes no nulos del álgebra A y desde ahora consideraremos, solamente las homótopas producidas por elementos de peso 1 .

Observación. Si $a \in I(\mathbf{A})$ entonces $a \in I\left(\mathbf{A}^{(a)}\right)$. En particular $\varepsilon \in I\left(\mathbf{A}^{(e)}\right)$.

\section{Resultados}

En esta sección consideraremos una T-álgebra $\mathbf{A}$ de rango 3, cuya t-ecuación es $x^{3}-(1+\gamma) \omega(x) x^{2}+\gamma \omega(x)^{2} x=0$ con $\gamma \neq \frac{1}{2}$, de dimensión finita sobre un cuerpo $\mathbf{K}$ y $\operatorname{car} \mathbf{K} \neq 2$. También supondremos que existe un elemento idempotente $\epsilon \in \mathbf{A}$ y escribiremos como $\mathbf{A}=\mathbf{K} e \oplus U \oplus V$, la descomposición de Peirce de $\mathbf{A}$ relativa a $f$, donde los subespacios $U$ y $V$ corresponden a

$$
i=\left\{x \in \operatorname{Ker} \omega \mid e x=\frac{1}{2}\right\}, V=\{x \in \operatorname{Ker} \omega / \epsilon x=\gamma x\}
$$


Es sabido desde [1] que en toda T-álgebra $\mathbf{A}$ de rango 3. se satisfacen las siguientes relaciones, entre los subespacios $U$ y $V$ :

$I^{2} \subset V, I V \subset I$ y $V^{2}=0$

Proposición 1. Sea $(\mathbf{A}, \omega)$ una $T$-álgebra de rango 3 y $a \in \mathbf{A}$. Si $b \in I\left(\mathbf{A}^{(a)}\right)$, entonces $\omega(b)=1$.

Demostración: Dado que $b \cdot{ }_{a} b=b$, sabemos que $\omega(b)^{2}=\omega(b)$ y en consecuencia $\omega(b)=0 \circ \omega(b)=1$.

Si $u(b)=0$, podemos escribir $b=u+v ; u \in U, v \in V$. Además consideremos $a=r+\bar{u}+\bar{v}$ con $\bar{u} \in U$ y $\bar{v} \in V$.

Rermplazando estas expresiones de $a$ y $b$ en la igualdad $b \cdot{ }_{a} b=b$ y usando algunas relacioness en T-álgebras de rango 3 mencionadas anteriormente, tenemos $u=-2 u^{2} \bar{u}+2 \gamma u v+4 v(u \bar{v})$ y $v=(1-\gamma) u^{2}+4 u(\bar{u} v)$.

Multiplicando la primera de estas ecuaciones por $v$ y la segunda por $u$, obtenemos $u^{2}(\bar{u} v)-2 u(u(\bar{u} v))=0$; pero de la identidad de Jacobi, sabemos que $2 u(u(\bar{u} v))=$ $-u^{2}(\bar{u} v)$, asi $u v=0$. Sustituyendo este valor de $u v$ en las expresiones obtenidas para $u$ y $v$, vemos que $u=-2 u^{2} \bar{u}$ y $v=(1-\gamma) u^{2}$.

De la igualdad $u=-2 u^{2} \bar{u}$ se demuestra que $u^{2}=0$ y en consecuencia $u=v=0$, es decir $b=0$.

De esta forma podemos concluir que $\omega(b)=1$ para todo elemento idempotente no nulo de $\mathbf{A}^{(a)}$.

Proposición 2. Sea $\mathbf{A}=\mathbf{K} \epsilon \oplus U \oplus V$ una $T$-álgebra de rango 3 con $\gamma \neq 1, a=$ $f+\bar{u}+\bar{v} ; \bar{u} \in U$ y $\bar{v} \in V$. Entonces

$I\left(\mathbf{A}^{(a)}\right)=\left\{f+u+\left[-\gamma \bar{v}+(1-\gamma)(1-2 \gamma)^{-1} u^{2}+2 \gamma(1-2 \gamma)^{-1} u \bar{u}\right]: u \in U\right\}$.

Demostración: Sea $f$ un idempotente no nulo en $\mathbf{A}^{(a)}$. Por Proposición 1, existen $u \in I^{*}$ y $v \in V$ tales que $f=\epsilon+u+v$. De la relación $f \cdot a f=f$, obtenemos $f+u+2(1-\gamma) \bar{u} v+2 \gamma u \bar{v}+2 \gamma u v+4 v(u \bar{v})-u^{2} \bar{u}+2 u(u \bar{u})+2 \gamma v+\gamma(2 \gamma-1) \bar{v}+$ $(1-\vartheta) u^{2}+2 \gamma u \bar{u}+4 u(\bar{u} v)=e+u+v$. Así vemos que se se satisfacen las dos igualdades siguientes:

$$
2(1-\gamma) \bar{u} v+2 \gamma u \bar{v}+2 \gamma u v+4 v(u \bar{v})-2 u^{2} \bar{u}=0
$$

$y$

$$
\gamma(2 \gamma-1) \bar{v}+(1-\gamma) u^{2}+2 \gamma u \bar{u}+4 u(\bar{u} v)=(1-2 \gamma) v
$$

Al multiplicar la igualdad (1) por $u$ y usando el hecho de que $\gamma \neq 1$ tenemos $u(u \bar{v})=0$.

Sustituyendo ese valor en la segunda igualdad concluimos que $v=-\gamma \bar{v}+(1-\gamma)(1-2 \gamma)^{-1} u^{2}+2 \gamma(1-2 \gamma)^{-1} u \bar{u}$

Corolario 1. Si la t-ecuación de $\mathbf{A}$ es $x^{3}-\omega(x) x^{2}=0$, entonces $I\left(\mathbf{A}^{(a)}\right)=I(\mathbf{A})$, para cualquier $a \in \mathbf{A}$ de peso 1 . 
Demostración: En este caso se tiene $\gamma=0$ y aplicando la Proposición 2 se tiene que $I\left(\mathbf{A}^{(a)}\right)=\left\{f+u+u^{2}: u \in I\right\}$.

Ouattara mostró que si la t-ecuación de $\mathbf{A}$ es $x^{3}-\omega(x) x^{2}=0$, entonces $\mathbf{A}$ es de Bernstein(ver [3]). Pero es sabido que los idempotentes en las álgebras de Bernstein son exactamente los elementos de la forma $f+u+u^{2}$.

En general los conjuntos $I(\mathbf{A})$ e $I\left(\mathbf{A}^{(a)}\right)$ no están ordenados por inclusión como muestra el siguiente ejemplo.

Ejemplo: Consideremos la T-álgebra de rango 3 y dimensión $3, \mathbf{A}=\langle\epsilon, u, v\rangle$, bajo la multiplic ación

$f^{2}=f, e u=\frac{1}{2} u, f v=v$ y los otros productos restantes nulos.

Se trata del caso $\gamma=1$. Haciendo $a=e+\imath$, notamos que el elemento $f=e-v$ satisface la igualdad $f{ }_{a} f=f$, pero $f^{2}=f \cdot f=e-2 v \neq f$, es decir $f \in I\left(\mathbf{A}^{(a)}\right)-I(\mathbf{A})$. Reciprocamente, el elemento $\epsilon+u$ es idempotente en $\mathbf{A}$; pero $\epsilon+u \notin I\left(\mathbf{A}^{(a)}\right)$, pues $(e+u) \cdot a(e+u)=e+u+v$.

Lema 1. Sea $\mathbf{A}$ una $T$-álgebra de rango 3 , entonces $\forall u, \bar{u} \in U$ y $v, \bar{v} \in V$ son válidas las siguientes igualdades:
(a) $u(\bar{v}(u v))=0$
(b) $u(\bar{u}(u v))=-(u \bar{u})(u v)$
(c) $\bar{u}(u(u \bar{u}))=0$
(d) $u(\bar{v}(u(u \bar{u})))=0$
(e) $v(\bar{u}(\bar{u}(u v)))=0$
(f) $v(\bar{v}(u(u \bar{u})))=(u \bar{v})(\bar{u}(u v))$ y $u(\bar{u}(\bar{v}(u v)))=(u \bar{v})(\bar{u}(u v))$
(g) $(u \bar{u})(\bar{v}(u v))=\bar{v}(v(u(u \bar{u}))$
(h) $\bar{v}(u(\bar{u}(u v)))=-u(\bar{u}(\bar{v}(u v)))$.

\section{Demostración:}

(a) $u\left(\bar{v}\left(u v^{\prime}\right)\right)=-(u v)(u \bar{v})$, por otra parte $u(\bar{v}(u v))=-u(v(u \bar{v}))=(u v)(u \bar{v})$. Luego $u(\bar{v}(u v))=0$.

(b) $u(\bar{u}(u v))+\bar{u}(u(u v))+(u \bar{u})(u v)=0$, pero $u(u v)=0$, entonces $u(\bar{u}(u v))=-(u \bar{u})(u v)$.

(c) $\bar{u}(u(u \bar{u}))=\bar{u}\left(-\frac{1}{2} u^{2} \bar{u}\right)=-\frac{1}{2} \bar{u}\left(\bar{u} u^{2}\right)=0$, pues $\bar{u} \in U$ y $u^{2} \in V$.

(d) $u\left(\bar{v}(u(u \bar{u}))=-(u \bar{v})(u(u \bar{u}))=(u \bar{v})\left(\frac{1}{2} u^{2} \bar{u}\right)=-\frac{1}{2} \bar{u}\left(u^{2}(u \bar{v})\right)=\right.$ $\frac{1}{2}\left(\bar{u}\left(\bar{v}\left(u^{2} u\right)\right)\right)=0$.

(e) $v(\bar{u}(\bar{u}(u v)))=-\frac{1}{2} v\left(\bar{u}^{2}(u v)\right)=\frac{1}{2} \bar{u}^{2}(v(u v))=0$. 
(f) $v^{\prime}(\bar{v}(u(u \bar{u})))=-\bar{v}(v((u(u \bar{u})))=\bar{v}((u \bar{u})(u v))=-\bar{v}(u(\bar{u}(u v)))$ por (b). Luego $v(\bar{v}(u(u \bar{u})))=(u \bar{v})(\bar{u}(u v))$. Por otro lado tenemos: $u\left(\bar{u}\left(\bar{v}\left(u v^{\prime}\right)\right)\right)=-u\left(\bar{u}(v(u \bar{v}))=u\left((u \bar{v})\left(\bar{u} v^{\prime}\right)\right)=-(u(\bar{u} v)(u \bar{v})=(u \bar{v})(\bar{u}(u v))\right.$.

(g) $(u \bar{u})\left(\bar{v}\left(u v^{\prime}\right)\right)=-\bar{v}\left(\left(u v^{\prime}\right)(u \bar{u})\right)=\bar{v}(v(u(u \bar{u})))$.

(h) $\bar{v}\left(u\left(\bar{u}\left(u v^{\prime}\right)\right)\right)=-(u \bar{v})(\bar{u}(u v))=-u(\bar{u}(\bar{v}(u v)))$ por $(\mathrm{f})$.

Proposición 3. Sea $\mathbf{A}$ una $T$-álgebra de rango 3 y $\mathbf{A}^{(a)}$ su álgebra homotópa, entonces: $x^{3}=0 \forall x \in$ Kerw, donde $x^{3}=(x \cdot a) \cdot_{a} x$.

Denlostración: Sean $a=\epsilon+\bar{u}+\bar{v}$ y $x=u+v \in$ fer $w$; con $u, \bar{u} \in U$ y $v, \bar{v} \in V$. Asi

$x^{2}=x \cdot a x=2(a x) x-a x^{2}=(1-\gamma) u^{2}+2 \gamma u v+4 u(u \bar{u})-4(u v) \bar{v}-4(u v) \bar{u} \mathrm{y}$

$r^{3}=\left\{\left[(1-\gamma) u^{2}+2 \gamma u v+4 u(u \bar{u})-4(u v) \bar{v}-4(u v) \bar{u}\right](\epsilon+\bar{u}+\bar{v})\right\}(u+v)+$

$\{(u+v)(\kappa+\bar{u}+\bar{v})\}\left[(1-\gamma) u^{2}+2 \gamma u v+4 u(u \bar{u})-4(u v) \bar{v}-4(u v) \bar{u}\right]-$

$(r+\bar{u}+\bar{v})\left\{\left[(1-\gamma) u^{2}+2 \gamma u v+4 u(u \bar{u})-4(u v) \bar{v}-4(u v) \bar{u}\right](u+v)\right\}$.

En el desarrollo del segundo miembro usamos las relaciones entre los subespacios $I^{\prime}, V$ (dadas en [1]) y resultados del Lema 1 anterior obteniendo lo que se quería, es decir $x^{3}=0$.

Proposición 4. Si A T-álgebra de rango 3 cuya homótopa también es una Tálgebra de rango 3 entonces $\mathbf{A}=\mathbf{K} \epsilon \in \mathrm{A} V$.

Demostración: Sea A una T-álgebra de rango 3. Si su homótopa $\mathbf{A}^{(a)}$ es también una T-álgehra de rango 3 , ella debe satisfacer una ecuación de la forma:

$$
x^{3}-(1+\delta) \omega(x) x^{2}+\delta \omega(x)^{2} x=0, \forall x \in \mathbf{A}
$$

Consideremos un elemento $x \in \mathbf{A}$ con $x=\alpha e+u+v$. Si $x^{2}=\alpha^{2} e+u^{\prime}+v^{\prime} \mathrm{y}$ $x^{3}=x^{3} f+u^{\prime \prime}+v^{\prime \prime}$, donde $x^{2}=x \cdot{ }_{a} x$ es el producto en la homótopa $\mathbf{A}$, entonces

$$
\alpha(1+\delta) u^{\prime}=u^{\prime \prime}+\alpha^{2} \delta u
$$

$y$

$$
\alpha(1+\delta) v^{\prime}=v^{\prime \prime}+\alpha^{2} \delta v
$$

En cálculo directo muestra que:

$u^{\prime}=\alpha[u+2 \gamma u \bar{v}+2(1-\gamma) \bar{u} v]+2 \gamma u v-4 \bar{v}(u v)-2 \bar{u}(u u)$.

$v^{\prime}=\alpha^{2} \gamma(2 \gamma-1) \bar{v}+2 \alpha \gamma(u \bar{u}+v)+(1-\gamma) u^{2}-4 \bar{u}(u v)$.

$u^{\prime \prime}=\frac{\alpha}{2}[u+2 \gamma u \bar{v}+2(1-\gamma) \bar{u} v]+\frac{\alpha^{2}}{2}\{[\alpha(u+2 \gamma u \bar{v}+2(1-\gamma) \bar{u} v)+2 \gamma u v-4 \bar{v}(u v)-$ $\left.2 \bar{u} u^{2}\right]+2 \gamma\left[\alpha(u+2 \gamma u \bar{v}+2(1-\gamma) \bar{u} v)+2 \gamma u v-4 \bar{v}(u v)-2 \bar{u} u^{2}\right] \bar{v}+2(1-\gamma) \bar{u}\left[\alpha^{2} \gamma(2 \gamma-\right.$ 1) $\left.\left.\bar{\tau}+2 \alpha \gamma(u \bar{u}+v)+(1-\gamma) u^{2}-4 \bar{u}(u v)\right]\right\}-2\left\{\bar{v}\left(u\left[\alpha^{2} \gamma(2 \gamma-1) \bar{v}+2 \alpha \gamma(u \bar{u}+v)+(1-\right.\right.\right.$ i) $\left.\left.u^{2}-4 \bar{u}(u v)\right]\right)+\bar{v}\left(\left[\alpha\left(u+2 \gamma u \bar{v}+2(1-\gamma) \bar{u} v+2 \gamma u v-4 \bar{v}(u v)-2 \bar{u} u^{2}\right] v\right)+\bar{u}(u[\alpha(u+\right.$ 
$\left.\left.\left.2 \gamma u \bar{v}+2(1-\gamma) \bar{u} v)+2 \gamma u v-4 \bar{v}(u v)-2 \bar{u} u^{2}\right]\right)\right\}+\gamma\left\{u\left[\alpha^{2} \gamma(2 \gamma-1) \bar{v}+2 n \gamma(u \bar{u}+v)+\right.\right.$

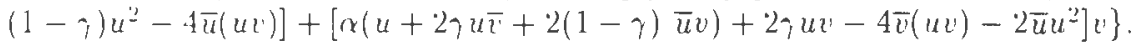

$i^{\prime \prime}=\alpha \alpha^{2} \gamma(2 \gamma-1) \bar{\gamma}+\alpha \gamma(u \bar{u}+v)+\alpha^{2} \gamma\{[\alpha(u+2 \gamma \bar{v}+2(1-\gamma) \bar{u} v)+2 \gamma u v-4 \bar{v}(u v)-$ $\left.\left.2 \bar{u} u^{2}\right] \bar{u}+\left[\alpha^{2} \gamma(2 \gamma-1) \bar{v}+2 \alpha \gamma(u \bar{u}+v)+(1-\gamma) u^{2}-4 \bar{u}(u v)\right]\right\}+(1-\gamma) u[\alpha \gamma(u+2 \gamma u \bar{v}+$ $\left.\left.\left.2(1-\gamma) \bar{u} v^{\prime}\right)+2 \gamma u v-4 \bar{v}(u v)-2 \bar{u} u^{2}\right)\right]-2\left\{\bar{u}\left(u\left[\alpha^{2} \gamma(2 \gamma-1) \bar{v}+2 \kappa \gamma(u \bar{u}+u)+\right.\right.\right.$ $\left.\left.\left.(1-\gamma) u^{2}-4 \bar{u}\left(u v^{\prime}\right)\right]\right)+\bar{u}\left(\left[\alpha(u+2 \gamma u \bar{v}+2(1-\gamma) \bar{u} v)+2 \gamma u v-4 \bar{v}(u v)-2 \bar{u} u^{2}\right] v^{\prime}\right)\right\}$.

Supongamos que $\gamma \neq 0$. Haciendo $x=$ xe y usando la ecuación (2) obtenemos (que $\alpha^{3} \gamma\left({ }^{2} 2 \gamma-1\right)(1+\delta) \bar{v}=\alpha^{3} \gamma \cdot(2 \gamma-1)(1+\alpha \gamma) \bar{v}$, es decir $\alpha^{3} \gamma(2 \gamma-1)(\delta-\alpha \gamma) \bar{v}=0$. Siendo $\gamma \neq 0$, podemos escoger $\alpha$ tal que $\delta-\alpha \gamma \neq 0$ y en tal caso $\bar{v}=0$. Para $x=\alpha r+u$ la ecuarión (1) indic a que $\frac{\alpha}{2}(\alpha-1)^{2} u+\left(2 \alpha \delta+\alpha^{2} \gamma^{2}-2 \alpha^{2} \gamma-\alpha \gamma^{2}\right) \bar{u} u^{2}-$ $\left(x^{3} \gamma(1-\gamma) u \bar{u}^{2}=0\right.$.

En particular si $u=\bar{u}$ entonces $\alpha(\alpha-1) \bar{u}=0$ de donde $\bar{u}=0$, luego $U=\{0\}$. Isando el hecho que $Y=\{0\}$ y la ecuación (2), se tiene que $\alpha(\alpha \delta-2 \alpha \gamma-2 \alpha \gamma \delta+$ $\left.\gamma+2 \alpha^{2} \gamma^{2}\right) v=0$, entonces $v=0$, de donde $V=\{0\}$.

Luego $\mathbf{A}=\mathbf{K} \boldsymbol{e}$ que es de rango 2. Contradicción con la hipótesis, luego es imposible que $\gamma \neq 0$.

Como $\gamma=0$, la t-ecuación de $\mathbf{A}$ es $x^{3}-\omega(x) x^{2}=0$. Para $x=\alpha \varepsilon+u$ la ecuación (1) indica que $\frac{\Omega}{2}(\alpha-1)^{2} u+2 \alpha \delta \bar{u} u^{2}=0$. Como esta igualdad es válida para todo $u \in L^{\prime}$, haciendo $u=\bar{u}$ tenemos que $\bar{u}=0 \mathrm{y}$ así $\alpha(\alpha-1)^{2} u=0$ de donde $u=0$. Luego $U=\{0\}$ y la ecuación (2) indica que $\alpha^{2} \delta v=0$ de donde $\delta=0$, salvo que $V=\{0\}$; pero el último caso se trata de $\mathbf{A}=\mathbf{K} f$ que es de rango 2. Luego $\mathbf{A}=\mathbf{K} f+V_{y}$ la álgebra homótopa es de la forma $\mathbf{A}^{(e+\bar{v})}$.

Recíprocamente, si $\mathbf{A}=\mathbf{K} e \forall$ tal que $x^{3}-\omega(x) x^{2}=0 \forall x \in \mathbf{A}$, entonces $x^{3}-x(x) x^{2}=0$.

\section{REFERENCIAS}

[1] Costa R., Principal train algebras of rank 3 and dimension $\leq 5$, Proc. Edinb. Math. Soc. 33, 61-70 (1990).

[2] Gonzalez S., Homotope Algcbra of Bernstcin Algebra, Submitted.

[3] Ouattara M.,Sur les T-algebres de Jordan, Linear Algebra and its Applications. 144,11-21(1991).

[4] Thedy A.Mutationen und polarisierte Fundamentalformel, Math. Annalen. 177, 235-246 (1968).

Recibido: Marzo 3, 1994.

Raúl Benavides y Rodolfo Baeza

Departamento de Matemáticas

Iniversidad de La Frontera

Casilla 54-D, Temuco-Chile. 